\title{
Accurate, Efficient, and Robust Q4-Like Membrane Elements Formulated in Cartesian Coordinates Using the Quasi-Conforming Element Technique
}

\author{
G. Shi, Y. Liu, and X. Wang \\ Department of Mechanics, Tianjin University, Tianjin 300072, China \\ Correspondence should be addressed to G. Shi; shi_guangyu@163.com
}

Received 19 September 2014; Revised 13 January 2015; Accepted 27 January 2015

Academic Editor: Chenfeng Li

Copyright (C) 2015 G. Shi et al. This is an open access article distributed under the Creative Commons Attribution License, which permits unrestricted use, distribution, and reproduction in any medium, provided the original work is properly cited.

\begin{abstract}
By using the quasi-conforming element technique, two four-node quadrilateral membrane elements with 2 degrees of freedom at each node (Q4-like membrane element) are formulated in rectangular Cartesian coordinates. One of the four-node quadrilateral membrane elements is based on the assumed strain field with only five independent strain parameters and accounting for the Poisson effect explicitly. There are no independent internal parameters and numerical integration involved in the evaluation of the strain parameters in these four-node quadrilateral membrane elements, and their element stiffness matrices are computed explicitly in Cartesian coordinates. Consequently, the formulation of these four-node quadrilateral membrane elements is extremely simple, and the resulting elements are very computationally efficient. These two quasi-conforming quadrilateral membrane elements pass the patch test and are free from shear locking and insensitive to the element distortion in the range of practical application. The numerical result comparison with other four-node quadrilateral membrane elements, including Q4-like plane elements with drilling degrees of freedom and the Q6-type isoparametric elements with very complicated nonconforming modes, shows that the present quasi-conforming quadrilateral membrane elements are not only reliable and robust, but also very accurate in both displacement and stress evaluations in the analysis of practical plane elasticity problems.
\end{abstract}

\section{Introduction}

The Q4-type membrane elements, which are the quadrilateral membrane elements with four corner nodes and only 2 degrees of freedom at each node as well no internal parameters involved, is one of the most useful finite elements since they are used not only in the displacement and stress analysis of membranes, but also in the four-node quadrilateral flatshell elements $[1,2]$. And four-node quadrilateral flat-shell elements are regarded as the most efficient shell element in the dynamic analysis involving surface contacts of panellike structures [3]. Because the original Q4 element in which each in-plane displacement component is approximated by a bilinear displacement interpolation in terms of four-nodal displacement variables suffers the shear locking when a membrane undergoes in-plane bending, Wilson et al. [4] pioneered the use of the higher-order displacement interpolations defined in terms of internal parameters to improve the performance of four-node quadrilateral membrane elements. The four-node quadrilateral membrane elements with two displacement-like internal parameters can be designated as Q6-type (or Q6-like) elements. The displacement interpolations in the original Q6 element proposed by Wilson et al. possesses nonconforming modes and Q6 fails to pass patch test although it can give good numerical results in many cases. Many researchers have devoted tremendous efforts to improve the properties and performance of Q6-type elements in the past five decades (vide the reference papers listed in [5]). And the employment of the nonconforming modes is still a major approach to improve the membrane elements based on assumed displacement fields up to now $[5,6]$.

The use of drilling degrees of freedom can efficiently remove the shear locking and improve the computational accuracy. Liu et al. [7] and Chen and Li [8] developed the quasi-conforming membrane elements with drilling degrees of freedom. Based on a functional treating drilling rotations 
as independent variables, Iura and Atluri [9] developed a reliable and accurate four-node membrane element with drilling degrees of freedom. Sze et al. [10] presented a fournode hybrid stress membrane element with drilling degrees of freedom. Some other papers on the membrane elements with drilling degrees of freedom using the conventional displacement approach can be found in [11]. These membrane elements with drilling degrees of freedom are more accurate indeed than the membrane elements with only translational degrees of freedom, but they are more computationally expensive since more nodal degrees of freedom are used.

Various hybrid stress methods and assumed strain methods have been used to develop improved membrane elements. Based on the earlier work, Pian and Sumihara [12] proposed a rational approach for assumed stress finite elements in 1983 and presented a Q4-like hybrid stress membrane element. In 1992, Sze et al. [10] used orthogonal stress modes in the fournode quadrilateral membrane elements with drilling degrees of freedom. The enhanced assumed strain method and the assumed natural strain methods are still been employed now by many researchers as an efficient scheme to remove shear locking in the membrane part of reliable and accurate shell elements $[6,13-15]$. The numerical integrations are used in all these improved membrane elements.

Although the development of four-node quadrilateral membrane elements based on various strategies has a long history and considerable achievements are accumulated, there is a renewed interest in recent years in the four-node quadrilateral membrane elements with the higher accuracy and computationally efficiency. Zhong and Ji [16] proposed a rational element approach to formulate displacement-based membrane elements. The so-called rational quadrilateral membrane element RQ4 is much better than Q4, but its computational accuracy is still not desirable enough. Cen and his coworkers $[5,17,18]$ presented a number of four-node quadrilateral membrane elements by using the quadrilateral area coordinates. Element AGQ6-I [17] which is based on the quadrilateral area coordinates exhibits excellent performance and it is quite insensitive to mesh distortions. However, AGQ6-I failed in the strict patch test and can only pass the weak patch test. Therefore, its convergence raised some discussions and several techniques were adopted to make it pass the patch test [5]. Unfortunately, the accuracy of the modified versions of AGQ6-I by all three different remedies deteriorates even though they can pass the strict patch test. The quadrilateral area coordinates are also employed to couple with the enhanced assumed strain method to derive reliable and accurate Q6-type membrane elements used in the flatshell element [6].

The quasi-conforming element technique proposed by Tang and his coworkers [19-22] is a general assumed strain method to formulate reliable and accurate elements. Although it has been primarily used for the developments of the quasiconforming plate and shell elements that involve with the $C^{1}$ continuity problems, a number of quasi-conforming membrane elements have been presented [2, 21-25]. Chen and Tang [21] presented a quasi-conforming quadrilateral isoparametric membrane element QC6 where two displacement-like internal parameters are used. Liu et al. [7] developed a quasiconforming membrane element with drilling degrees of freedom QR4 in which the isoparametric element technique was also used. Based on the quasi-conforming element technique, Chen and $\mathrm{Li}$ [8] presented some improved quadrilateral membrane elements with drilling degrees of freedom also in terms of the natural coordinates. Numerical integration is used in the elements given by Liu et al. [7] as well as by Chen and $\mathrm{Li}[8]$. Based on the assumed element strains given by the rational displacement field, Liu et al. [26] presented a quasiconforming quadrilateral membrane element AQCE4 in the Cartesian coordinates. AQCE4 yields very good results when the coarse meshes are used, but it seems not to converge to the exact solution as the mesh is getting finer.

As a matter of fact, by using the assumed strain method in the quasi-conforming element technique, Shi and Voyiadjis derived a four-node quadrilateral cylindrical membrane element for the four-node cylindrical shell elements in 1991 [23, 27] and a four-node quadrilateral plane element for the membrane part of a four-node nonlinear quadrilateral flat-shell element in Cartesian coordinates in 1991 [2]. The element stiffness matrices of both the quadrilateral cylindrical shell element and the flat shell are evaluated explicitly. However, the performance of this four-node quadrilateral membrane element when it is used alone was not reported since the focus of these shell elements is the flexural analysis of shells.

The computational efficiency is a very critical issue in nonlinear and dynamic analysis of structures. The reliable one point quadrature is believed to be efficient for both membrane and shell elements (please refer to the related references given in [6]). Then, the quadrilateral membrane elements with explicit element stiffness, that is, there are no any numerical integrations, could be more computationally efficient and desirable. Based on the quasi-conforming element technique and the strain field derived from the displacement interpolation, Xia et al. [28] presented two quasi-conforming quadrilateral membrane elements WDC4 and WDC6 where two displacement-like internal parameters are utilized in WDC6. The element stiffness matrices of both WDC4 and WDC6 are evaluated explicitly in Cartesian coordinates. WDC4 and WDC6 pass the patch test and are very efficient. Thus it was claimed that WDC4 and WDC6 are the first quadrilateral membrane elements directly formulated in Cartesian coordinates that are able to pass patch test. Unfortunately, the aforementioned claim about the quadrilateral membrane elements formulated in Cartesian coordinates is incorrect. Furthermore, the accuracy of WDC4 and WDC6 are not competitive to other quasi-conforming quadrilateral membrane elements reported in the literature such as AQCE4 [26]. The details about the accuracy comparison will be shown later.

The objective of this paper is to develop a more accurate four-node quadrilateral quasi-conforming membrane element with two degrees of freedom per node by explicitly taking account of the Poisson effect in the assumed element strain field. This new four-node quadrilateral quasiconforming membrane element should be not only reliable, but also more computationally efficient than the improved four-node quadrilateral membrane elements based on other methods. The performance of the four-node quadrilateral 


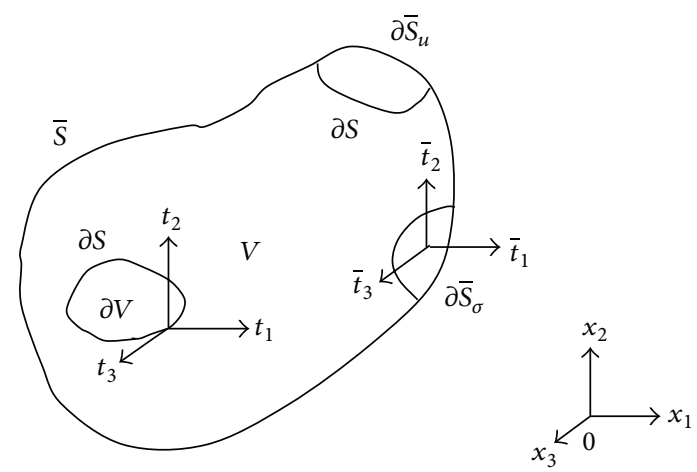

Figure 1: A typical continuum with various types of boundaries.

assumed strain membrane element developed in 1990 for the membrane part of a four-node quadrilateral flat-shell element [2] is also presented here for the first time. Both of these fournode quadrilateral quasi-conforming membrane elements pass the patch test, exhibit no shear locking, and are insensitive to the element distortion in the range of practical application. The numerical result comparison with other fournode quadrilateral membrane elements, including both Q4like elements with drilling degrees of freedoms and Q6-type elements, indicates that the four-node quadrilateral quasiconforming membrane elements presented in this paper are not only reliable and robust but also very accurate indeed.

\section{General Formulation of Quasi-Conforming Elements}

A typical continuum considered in the boundary value problem is depicted in Figure 1, where $x_{i}(i=1,2,3)$ denote the rectangular Cartesian coordinates, $\bar{S}_{\sigma}$ and $\bar{S}_{u}$ represent, respectively, the force boundary and the displacement boundary on $\bar{S}$, respectively, $\bar{t}_{i}$ are the given tractions on $\bar{S}_{\sigma}$, $n_{j}(j=1,2,3)$ are used to represent the direction cosines of a point on $\bar{S}_{\sigma}$, and $\partial V$ and $\partial S$ signify, respectively, a subdomain and its surface in the continuum.

By following the notations used in theory of elasticity, the displacement, strain, stress fields, and body forces can be expressed, respectively, as $u_{i}, \varepsilon_{i j}, \sigma_{i j}$, and $f_{i}$ with $i, j=1,2,3$. Theory of elasticity gives the governing equations of a body with domain $V$ and boundary $\bar{S}$ illustrated in Figure 1 as

$$
\begin{gathered}
\frac{\partial \sigma_{i j}}{\partial x_{j}}+f_{i}=0 \quad \text { in } V, \\
\varepsilon_{i j}=\frac{1}{2}\left(\frac{\partial u_{i}}{\partial x_{j}}+\frac{\partial u_{j}}{\partial x_{i}}\right) \quad \text { in } V, \\
\sigma_{i j}=\frac{\partial U}{\partial \varepsilon_{i j}} \text { or } \varepsilon_{i j}=\frac{\partial W}{\partial \sigma_{i j}} \quad \text { in } V, \\
\sigma_{i j} n_{j}=\bar{t}_{i} \text { on } \bar{S}_{\sigma}, \\
u_{i}=\bar{u}_{i} \text { on } \bar{S}_{u},
\end{gathered}
$$

where $U\left(\varepsilon_{i j}\right)$ denotes the strain energy density in terms of strains $\varepsilon_{i j}$, and $W\left(\sigma_{i j}\right)$ denotes the complementary energy density in terms of stresses $\sigma_{i j}$. These governing equations of elasticity are satisfied in the weak form in the finite element method. Although the strain energy density $U$ and complementary energy density $W$ are identical in the case of linear deformations of isotropic materials, they have different independent variables. The choice of the independent variables is very critical in the multifield finite element formulations.

The conventional finite elements method is based on the trial displacement field only, where any admissible trial displacement field is required to satisfy the conforming condition along the element interfaces. The element stiffness matrix of a conventional finite element can be evaluated from the minimum potential principle or other types of weak forms of equilibrium equations in terms of the trial displacements. Unfortunately, the formulation of displacement-based finite elements encounters the difficulty to satisfy the conforming condition. For example, the simplest four-node quadrilateral membrane element Q4 formulated in the rectangular Cartesian coordinates of the element fails to satisfy the conforming condition even though the four-node rectangular membrane element is lucky to meet the conforming condition. One way to expand the admissible trial functions is to adopt the multifield formulation $[19,22]$. If one chooses both the displacements $u_{i}$ and independent strains $\widetilde{\varepsilon}_{i j}$ as the trial functions, the strain-displacement relation defined in (2) is not satisfied $a$ priori. Then both the generalized equilibrium and the generalized compatibility have to be enforced in the weak form [29]. If one uses $\delta \sigma_{i j}$ to denote the test functions (or called as weighting functions) for the compatibility condition, one has [29]

$$
\begin{aligned}
& \delta\left\{\iiint_{V}\left[U\left(\widetilde{\varepsilon}_{i j}\right)-f_{i} u_{i}\right] d v-\iint_{\bar{S}_{\sigma}} \bar{t}_{i} u_{i} d s\right\}=0, \\
& (i, j=1,2,3), \\
& \iiint_{V}\left[\left(\frac{\partial u_{i}}{\partial x_{j}}+\frac{\partial u_{j}}{\partial x_{i}}\right)-2 \widetilde{\varepsilon}_{i j}\right] \delta \sigma_{i j} d v \\
& \quad+\iint_{\bar{S}_{u}}\left[\left(\bar{u}_{i}-u_{i}\right) n_{i}+\left(\bar{u}_{j}-u_{j}\right) n_{j}\right] \delta \sigma_{i j} d s=0 .
\end{aligned}
$$

It should be noticed that the strain energy density $U$ in (6) is in terms of independent trial strains $\widetilde{\varepsilon}_{i j}$ rather than the strains derived from the assumed displacement field.

In the finite element analysis, a continuum is discretized into a finite number of elements. Let $M$ be the number of total elements in the continuum under consideration, and then the weak forms of generalized equilibrium and compatibility equations for a continuum discretized by finite element method take the form

$$
\begin{array}{r}
\delta\left\{\sum_{e=1}^{M} \iiint_{\partial V_{e}}\left[U_{e}\left(\widetilde{\varepsilon}_{i j}\right)-f_{i} u_{i}\right] d v-\iint_{\bar{S}_{\sigma}} \bar{t}_{i} u_{i} d s\right\}=0, \\
(i, j=1,2,3),
\end{array}
$$




$$
\begin{aligned}
& \sum_{e=1}^{M} \iiint_{\partial V_{e}}\left[\left(\frac{\partial u_{i}}{\partial x_{j}}+\frac{\partial u_{j}}{\partial x_{i}}\right)-2 \widetilde{\varepsilon}_{i j}\right] \delta \sigma_{i j} d v \\
& \quad+\sum_{e b=1}^{N_{b}} \iint_{\partial S_{e b}}\left[\left(u_{i}^{+}-u_{i}^{-}\right) n_{i}+\left(u_{j}^{+}-u_{j}^{-}\right) n_{j}\right] \delta \sigma_{i j} d s \\
& \quad+\iint_{\partial \bar{s}_{u}}\left[\left(\bar{u}_{i}-u_{i}\right) n_{i}+\left(\bar{u}_{j}-u_{j}\right) n_{j}\right] \delta \sigma_{i j} d s=0,
\end{aligned}
$$

where $N_{b}$ is the number of common surfaces/boundaries between elements and the superscripts "+" and "-" signify the displacements associated with different elements along a common surface/boundary. In fact, the second term in the weak form of generalized compatibility equation for a continuum discretized by the finite element method specified in (9) denotes the weak form of the displacement conforming conditions (also called as compatible conditions) between the neighboring elements, and the last term in (9) is the weak form of specified displacement boundary conditions which can be satisfied easily in the finite element method. Since the strain-displacement relations and the conforming condition along the element boundaries are satisfied in a weighted integral form as shown in (9) instead of the point-wise compatibility condition, the trial function of displacement field used in a finite element meeting the compatibility characterized in (9) is said satisfying the quasi-conforming condition. Therefore, the physical meaning of (9) is the quasi-conforming condition in the displacement-based finite elements.

When the displacement conforming condition between the elements is set to be satisfied a priori, which is the case in the element formulation of the quasi-conforming elements, and the displacement boundary conditions are enforced posteriori, then (9) reduces to the following form:

$$
\sum_{e=1}^{M} \iiint_{\partial V_{e}}\left[\left(\frac{\partial u_{i}}{\partial x_{j}}+\frac{\partial u_{j}}{\partial x_{i}}\right)-2 \widetilde{\varepsilon}_{i j}\right] \delta \sigma_{i j} d v=0
$$

If the assumed strains $\widetilde{\varepsilon}_{i j}$ are approximated independently over each element, the weak form of the strain-displacement relation given in the equation above can be enforced in each element individually as

$$
\iiint_{\partial V_{e}}\left[\left(\frac{\partial u_{i}}{\partial x_{j}}+\frac{\partial u_{j}}{\partial x_{i}}\right)-2 \widetilde{\varepsilon}_{i j}\right] \delta \sigma_{i j} d v=0 .
$$

Equation (11) provides the basis for the quasi-conforming element technique $[19,29]$, although the Hu-Washizu variational principle can also be used for the quasi-conforming element technique [19].

For a linear elastic continuum, the strain energy density in terms of the trial strains $\widetilde{\varepsilon}_{i j}$, assumed independently over an element, $U\left(\widetilde{\varepsilon}_{i j}\right)$, can be expressed as

$$
U\left(\widetilde{\varepsilon}_{i j}\right)=\frac{1}{2} \widetilde{\varepsilon}_{i j} C_{i j k l} \widetilde{\varepsilon}_{k l}, \quad(i, j, k, l=1,2,3)
$$

in which $C_{i j k l}$ is the components of elastic tensor of the continuum. Then it follows from (8) and (12) that the strain

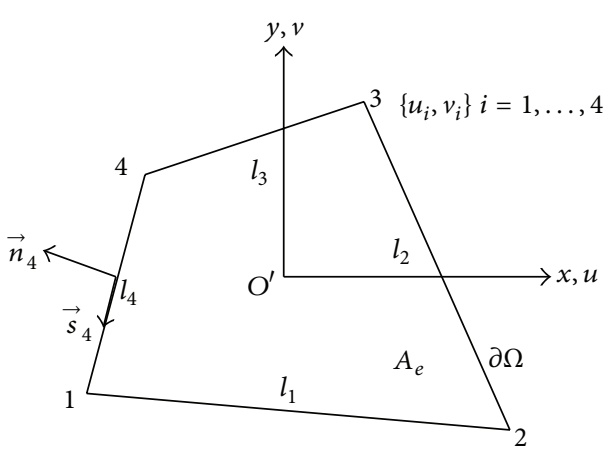

FIGURE 2: A typical quadrilateral membrane element with four corner nodes.

energy over a subdomain (an element) $\pi_{e}$ can be expressed in terms of the element stiffness matrix given by the quasiconforming element technique as

$$
\pi_{e}=\frac{1}{2} \iiint_{\partial V_{e}} U\left(\widetilde{\varepsilon}_{i j}\right) d v=\frac{1}{2} \iiint_{\partial V_{e}} \widetilde{\varepsilon}_{i j} C_{i j k l} \widetilde{\varepsilon}_{k l} d v=\frac{1}{2} \mathbf{q}^{T} \mathbf{K}_{e} \mathbf{q}
$$

in which $\mathbf{K}_{e}$ is the element stiffness matrix and $\mathbf{q}$ is the nodal displacement vector of the element under consideration.

A number of the assumed strain elements based on the quasi-conforming element technique have been developed $[2,19-25,27]$. The numerical examples show that the quasiconforming element technique could not only solve the conforming difficulties in plate elements, but also yield very accurate and efficient assumed strain elements.

\section{QCQ4-1: A Simple Quadrilateral Quasi-Conforming Membrane Element}

The formulation of Quasi-Conforming Quadrilateral (4 nodes) membrane element, named as QCQ4-1, is briefly presented in this section. As mentioned in Introduction, this membrane element was originally developed for the membrane part of a simple and accurate four-node quadrilateral flat-shell element $[2,23,27]$. It is still worthwhile to present the element formulation here since no people really know it well as its detailed formulation and the performance used alone have been never reported in the literature [28]. And moreover, even the first author of the papers $[2,23]$ was not aware of the value of this four-node quadrilateral quasiconforming membrane element until he read several recently published papers on the formulation of efficient quadrilateral membrane elements.

3.1. The Definition of the Element Local Coordinates. The topology and nodal degrees of freedom of four-node quadrilateral membrane element QCQ4-1 are depicted in Figure 2, where each node has two translational degrees of freedom. The origin of the element local coordinates is chosen to coincide with the element centroid, so that the static moments 
with respect to the element local coordinates over the element are vanished; that is,

$$
\iint_{A_{e}} x d x d y=0, \quad \iint_{A_{e}} y d x d y=0,
$$

where $A_{e}$ is the area of the element under consideration.

The nodal displacement vector of a four-node quadrilateral element illustrated in Figure 2 takes the following form:

$$
\mathbf{q}=\left\{\begin{array}{llllllll}
u_{1} & v_{1} & u_{2} & v_{2} & u_{3} & v_{3} & u_{4} & v_{4}
\end{array}\right\}^{T} .
$$

Each edge of the element has a unit outward normal vector defined by the direction cosines as

$$
\mathbf{n}_{i}=\left\{n_{x i}, n_{y i}\right\}^{T}=\left\{\cos \left(\vec{n}_{i}, x\right), \cos \left(\vec{n}_{i}, y\right)\right\}^{T} .
$$

3.2. The Assumed Strain Field for QCQ4-1. It is more convenient to use the matrix form to formulate the element stiffness matrix instead of the equations in terms of tensors given in the previous section. The nonzero strain components in a membrane element defined in the $x-y$ plane as illustrated in Figure 2 take the form

$$
\boldsymbol{\varepsilon}=\left\{\begin{array}{c}
\varepsilon_{x} \\
\varepsilon_{y} \\
2 \varepsilon_{x y}
\end{array}\right\}=\left\{\begin{array}{c}
\frac{\partial u}{\partial x} \\
\frac{\partial v}{\partial y} \\
\frac{\partial u}{\partial y}+\frac{\partial v}{\partial x}
\end{array}\right\} .
$$

The assumed strains in the four-node quadrilateral quasiconforming membrane element QCQ4-1 proposed by Shi and Voyiadjis $[2,23]$ are of the form

$$
\widetilde{\boldsymbol{\varepsilon}}=\left\{\begin{array}{c}
\widetilde{\varepsilon}_{x} \\
\widetilde{\varepsilon}_{y} \\
2 \widetilde{\varepsilon}_{x y}
\end{array}\right\}=\left[\begin{array}{lllll}
1 & y & 0 & 0 & 0 \\
0 & 0 & 1 & x & 0 \\
0 & 0 & 0 & 0 & 1
\end{array}\right]\left\{\begin{array}{c}
\alpha_{1} \\
\vdots \\
\alpha_{5}
\end{array}\right\}=\mathbf{P}_{1} \boldsymbol{\alpha},
$$

where $\mathbf{P}_{1}$ is the interpolation matrix for the assumed element strain field and $\alpha_{i}(i=1,2,3,4,5)$ are the corresponding generalized strain parameters which will be determined in terms of the element nodal displacements later. The five strain parameters in (18) are independent of each other so that the assumed element strain field given in (18) can meet the rank requirements of element stiffness matrix $[19,20]$.

As shown in (18), the strain interpolation used in this paper is not a complete linear polynomial. However, such a strain interpolation with five parameters theoretically satisfies the criterion of the coordinate invariance as this strain interpolation is corresponding to the displacement interpolation in the form of a complete quadratic polynomial with a zero value of Poisson ratio given in (41) and (42). Of course, the coordinate invariant of the strain interpolation defined in (18) will be verified by numerical examples later.
3.3. The Determination of the Generalized Strain Parameters. The quasi-conforming condition given in (11) is employed to determine the generalized strain parameters in (18). By taking $\mathbf{P}_{1}$ as the test function used in (11), the quasi-conforming condition for the assumed strains given in (18) takes the form

$$
\iint_{A_{e}} \mathbf{P}_{\mathbf{1}}{ }^{T} \boldsymbol{\varepsilon} d x d y=\iint_{A_{e}} \mathbf{P}_{\mathbf{1}}{ }^{T} \widetilde{\boldsymbol{\varepsilon}} d x d y=\iint_{A_{e}} \mathbf{P}_{\mathbf{1}}{ }^{T} \mathbf{P}_{\mathbf{1}} \boldsymbol{\alpha} d x d y .
$$

The integral of the left hand side of this equation could be degenerated into the integration along the element edges by using integration by parts. For instance, by substituting (17) into (19), one obtains the first line of (19) as

$$
\iint_{A_{e}} \frac{\partial u}{\partial x} d x d y=\iint_{A_{e}} \widetilde{\varepsilon}_{x} d x d y=\iint_{A_{e}}\left(\alpha_{1}+\alpha_{2} y\right) d x d y .
$$

The left hand side of the equation above becomes

$$
\iint_{A_{e}} \frac{\partial u}{\partial x} d x d y=\oint_{\partial \Omega} u n_{x} d s=\sum_{i=1}^{4} \int_{l_{i}} u n_{x i} d s_{i}
$$

in which $l_{i}$ is the length of the $i$ th edge of the element and $n_{x i}=\cos \left(\vec{n}_{i}, x\right)$ is associated with the direction cosines of the outward normal of the $i$ th edge of the element. The displacement $u(s)$ along each edge of the element can be interpolated by a linear function in terms of the nodal displacement parameters at the two nodes of the element edge. Then the integration along the edges of the element can be computed explicitly as

$$
\begin{aligned}
& \iint_{A_{e}} \frac{\partial u}{\partial x} d x d y \\
& \quad=\left[\begin{array}{lllllllll}
C_{11} & C_{12} & C_{13} & C_{14} & C_{15} & C_{16} & C_{17} & C_{18}
\end{array}\right] \mathbf{q}=\mathbf{C}_{1} \mathbf{q},
\end{aligned}
$$

where $C_{1 j}(j=1,2, \ldots, 8)$ is associated only with the element edge lengths and the direction cosines of the outward normal of the element edges, $\mathbf{q}$ is the element nodal displacement vector defined in (15), and $\mathbf{C}_{\mathbf{1}}$ is the first row of matrix $\mathbf{C}$ which will be explained later.

By using (14), the last integral in (20) leads to

$$
\iint_{A_{e}}\left(\alpha_{1}+\alpha_{2} y\right) d x d y=\alpha_{1} \iint_{A_{e}} d x d y=\alpha_{1} A_{e} .
$$

Then the generalized strain parameter $\alpha_{1}$ defined in (18) is expressed by the nodal displacement vector $\mathbf{q}$ of the element as

$$
\alpha_{1}=\frac{1}{A_{e}} \mathbf{C}_{\mathbf{1}} \mathbf{q}
$$

The process to evaluate the generalized strain parameter $\alpha_{2}$ is in the same manner. The second row of (19), which 
is obtained by taking the second column of $\mathbf{P}_{1}$ as the test function, takes the form

$$
\begin{aligned}
\iint_{A_{e}} y \frac{\partial u}{\partial x} d x d y & =\iint_{A_{e}} y \widetilde{\varepsilon}_{x} d x d y \\
& =\iint_{A_{e}} y\left(\alpha_{1}+\alpha_{2} y\right) d x d y \\
& =\alpha_{2} \iint_{A_{e}} y^{2} d x d y .
\end{aligned}
$$

The first integral in the above equation can be written as

$$
\iint_{A_{e}} y \frac{\partial u}{\partial x} d x d y=\oint_{\partial \Omega} y u n_{x} d x=\sum_{i=1}^{4} \int_{l_{i}} y u n_{x i} d s_{i} .
$$

Then, the integration along the edges of a four-node membrane element depicted in Figure 2 can be expressed by the nodal displacements vector $\mathbf{q}$ defined in (15) as follows:

$$
\begin{aligned}
& \iint_{A_{e}} y \frac{\partial u}{\partial x} d x d y \\
& \quad=\left[\begin{array}{llllllllll}
C_{21} & C_{22} & C_{23} & C_{24} & C_{25} & C_{26} & C_{27} & C_{28}
\end{array}\right] \mathbf{q}=\mathbf{C}_{2} \mathbf{q} .
\end{aligned}
$$

Thus $\alpha_{2}$ is determined in terms of the nodal displacements vector $\mathbf{q}$ as

$$
\alpha_{2}=\frac{1}{\iint_{A_{e}} y^{2} d x d y} \mathbf{C}_{2} \mathbf{q} .
$$

The other strain parameters can be determined in the same way $[19,24]$. The whole process to determine the strain parameters based on (19) can be expressed in the matrix form as

$$
\begin{gathered}
\iint_{A_{e}} \mathbf{P}_{\mathbf{1}}{ }^{T} \varepsilon d x d y=\mathbf{C} \mathbf{}, \\
\iint_{A_{e}} \mathbf{P}_{\mathbf{1}}{ }^{T} \mathbf{P}_{\mathbf{1}} \boldsymbol{\alpha} d x d y=\iint_{A_{e}} \mathbf{P}_{\mathbf{1}}{ }^{T} \mathbf{P}_{\mathbf{1}} d x d y \boldsymbol{\alpha}=\mathbf{A} \boldsymbol{\alpha},
\end{gathered}
$$

where $\mathbf{C}$ is a $5 \times 8$ matrix, $\mathbf{A}$ is a $5 \times 5$ matrix, and $\mathbf{q}$ is the nodal displacement vector of the four-node membrane element defined in (15).

The explicit form of matrix $\mathbf{A}$ can be evaluated in the following form:

$$
\begin{aligned}
\mathbf{A} & =\iint_{A_{e}}\left[\begin{array}{lllll}
1 & y & 0 & 0 & 0 \\
y & y^{2} & 0 & 0 & 0 \\
0 & 0 & 1 & x & 0 \\
0 & 0 & x & x^{2} & 0 \\
0 & 0 & 0 & 0 & 1
\end{array}\right] d x d y \\
& =\left[\begin{array}{ccccc}
A_{e} & & & & \\
& J_{x} & & \mathbf{0} & \\
& & A_{e} & & \\
& 0 & & J_{y} & \\
& & & & A_{e}
\end{array}\right],
\end{aligned}
$$

where (14) is used, $A_{e}$ is the area of the quadrilateral element depicted in Figure 2, and $J_{x}$ and $J_{y}$ are of the form

$$
\begin{aligned}
& J_{x}=\iint_{A_{e}} y^{2} d x d y, \\
& J_{y}=\iint_{A_{e}} x^{2} d x d y .
\end{aligned}
$$

The integrals in the equations above can be carried out easily in the element local Cartesian coordinates depicted in Figure 2. Therefore, $\mathbf{A}$ will be a diagonal matrix if the origin of the element local coordinates is chosen at the element centroid.

By substituting (29) into (19), one can determine the element strain parameters $\boldsymbol{\alpha}$ explicitly in terms of element nodal displacement vector $\mathbf{q}$ as

$$
\boldsymbol{\alpha}=\mathbf{A}^{-1} \mathbf{C q}
$$

where $\mathbf{A}^{-1}$, the inverse of $\mathbf{A}$, in the equation above can be computed easily because it is a diagonal matrix as shown in (30). $\mathrm{A}^{-1}$ takes the form

$$
\mathbf{A}^{-1}=\left[\begin{array}{cccccc}
\frac{1}{A_{e}} & & & & \\
& \frac{1}{J_{x}} & & & 0 & \\
& & \frac{1}{A_{e}} & & \\
& 0 & & \frac{1}{J_{y}} & \\
& & & & \frac{1}{A_{e}}
\end{array}\right] .
$$

Then it follows from (18) and (32) that the interpolated element strain field is expressed in terms of element nodal displacement vector $\mathbf{q}$ as

$$
\widetilde{\varepsilon}=\mathbf{P}_{1} \alpha=\mathbf{P}_{1} \mathbf{A}^{-1} \mathbf{C q} .
$$

The interpolated element strain field given in the equation above can be also written in the standard form used in the conventional finite element method as

$$
\widetilde{\varepsilon}=\mathbf{B q},
$$

where matrix $\mathbf{B}$ takes the form

$$
\mathbf{B}=\mathbf{P}_{1} \mathbf{A}^{-1} \mathbf{C} .
$$

3.4. The Evaluation of Element Stiffness Matrix in QCQ4-1. The general form of the element stiffness matrix is given in (13). When the element strains in plane stress problems are expressed in the vector form defined in (19), the elastic tensor corresponding to $C_{i j k l}$ in (13) can be replaced by the elastic matrix $\mathbf{D}$ for plane stress problems as

$$
\mathbf{D}=\frac{E}{1-\mu^{2}}\left[\begin{array}{ccc}
1 & \mu & 0 \\
\mu & 1 & 0 \\
0 & 0 & \frac{1-\mu}{2}
\end{array}\right]
$$


in which $E$ is the Yong's modulus and $\mu$ is Poisson ratio of the material under consideration. By substituting (34) and (37) into (13), one has

$$
\iint_{A_{e}} \widetilde{\boldsymbol{\varepsilon}}^{\mathbf{T}} \mathbf{D} \widetilde{\boldsymbol{\varepsilon}} d x d y=\mathbf{q}^{T} \mathbf{K}_{\mathbf{e}} \mathbf{q} .
$$

Then the element stiffness matrix in a quasi-conforming membrane element with area $A_{e}$ is of the form

$$
\mathbf{K}_{\mathbf{e}}=\iint_{A_{e}} \mathbf{C}^{\mathrm{T}} \mathbf{A}^{-1} \mathbf{P}_{\mathbf{1}}^{\mathrm{T}} \mathbf{D} \mathbf{P}_{\mathbf{1}} \mathbf{A}^{-1} \mathbf{C} d x d y .
$$

Because the matrices $\mathbf{A}$ and $\mathbf{C}$ are independent of $x$ and $y$, so that the element stiffness matrix $\mathbf{K}_{e}$ becomes

$$
\mathbf{K}_{\mathbf{e}}=\mathbf{C}^{T} \mathbf{A}^{-1} \iint_{A_{e}} \mathbf{P}_{\mathbf{1}}^{T} \mathbf{D} \mathbf{P}_{\mathbf{1}} d x d y \mathbf{A}^{-1} \mathbf{C}
$$

It should be noticed that the element stiffness matrix in the expression above is evaluated in the element local Cartesian coordinates. As shown in (18), $\mathbf{K}_{e}$ is an interpolation matrix composed of simple polynomial; therefore the element stiffness matrix $\mathbf{K}_{e}$ defined in (40) can be computed explicitly in Cartesian coordinates. Consequently, element QCQ4-1 possesses an explicit element stiffness matrix.

\section{QCQ4-2: A Four-Node Quadrilateral Quasi-Conforming Membrane Element Accounting for the Poisson Effect}

This section presents the formulation of Quasi-Conforming Quadrilateral (4 nodes) membrane element accounting for the Poisson effect which is designated as QCQ4-2 for convenience.

QCQ4-2 has the same element topology and nodal displacement degrees of freedom as those of QCQ4-1 which is illustrated in Figure 2. The element local coordinates are also established in the same manner as QCQ4-1.

4.1. The Two-Dimensional Analytical Displacement Field Consistent with Theory of Elasticity. In 1996, Zhong and Ji [16] proposed to use the so-called rational displacement trial function for the displacement interpolation of the displacement-based membrane finite elements. The rational displacement trial function for a membrane element is the displacement field consistent with the analytical solution of the plane stress problem in theory of elasticity. A displacement trial field for a four-node quadrilateral membrane element takes the following form [16]:

$$
\begin{aligned}
u= & a_{1}+0+a_{3} y+a_{4} x-a_{5} \mu x+\frac{1}{2} a_{6} y \\
& -a_{7} x y+\frac{1}{2} a_{8}\left(\mu x^{2}+y^{2}\right), \\
v= & 0+a_{2}-a_{3} x-a_{4} \mu y+a_{5} y \\
& +\frac{1}{2} a_{6} x+\frac{1}{2} a_{7}\left(x^{2}+\mu y^{2}\right)-a_{8} x y,
\end{aligned}
$$

where $a_{i}(i=1,2, \ldots, 8)$ are the general displacement parameters and $\mu$ is Poisson ratio. It can be seen that the Poisson effect is included in the so-called rational displacement field defined in (41) and (42).

4.2. The Assumed Strain Field Accounting for Poisson Effect. It follows from (41) and (42) that the assumed strain field for a four-node membrane element consistent with the rational displacement field of plane elasticity problems is of the form

$$
\left\{\begin{array}{c}
\widetilde{\varepsilon}_{x} \\
\widetilde{\varepsilon}_{y} \\
2 \widetilde{\varepsilon}_{x y}
\end{array}\right\}=\left[\begin{array}{ccccc}
1 & y & 0 & -\mu x & 0 \\
0 & -\mu y & 1 & x & 0 \\
0 & 0 & 0 & 0 & 1
\end{array}\right]\left\{\begin{array}{c}
\alpha_{1} \\
\vdots \\
\alpha_{5}
\end{array}\right\}=\mathbf{P}_{2} \boldsymbol{\alpha}
$$

where $\mathbf{P}_{2}$ is the interpolation matrix of the assumed element strain field and $\alpha_{i}(i=1,2,3,4,5)$ are the generalized strain parameters.

The strain filed given in (43) is used for the four-node quadrilateral membrane element QCQ4-2. One can see that the assumed strain field in QCQ4-2 also has only five independent generalized strain parameters as that in QCQ41. Equation (43) shows that the Poisson effect is taken into account by the generalized strain parameters $\alpha_{2}$ and $\alpha_{4}$ in QCQ4-2.

Equation (43) indicates that the assumed element strain field with a constant shear strain in QCQ4-1 which was first used by Shi and Voyiadjis in 1991 [23] is consistent with the so-called rational displacement trial function for a four-node quadrilateral membrane element.

4.3. The Element Stiffness Matrix of QCQ4-2. Because QCQ42 has the same element nodal displacements as QCQ4-1, then the generalized strain parameters in (43) can be determined in the same manner as that used for QCQ4-1. The matrix C derived for the formulation of QCQ4-1 can be used for QCQ4-2. By following the procedure used for (32), the assumed element strains in QCQ4-2 can be expressed in terms of element nodal displacement vector $\mathbf{q}$ as

$$
\left\{\begin{array}{c}
\widetilde{\varepsilon}_{x} \\
\widetilde{\varepsilon}_{y} \\
2 \widetilde{\varepsilon}_{x y}
\end{array}\right\}=\left[\begin{array}{ccccc}
1 & y & 0 & -\mu x & 0 \\
0 & -\mu y & 1 & x & 0 \\
0 & 0 & 0 & 0 & 1
\end{array}\right]\left\{\begin{array}{c}
\alpha_{1} \\
\vdots \\
\alpha_{5}
\end{array}\right\}=\mathbf{P}_{2} \mathbf{A}_{2}{ }^{-1} \mathbf{C q}
$$

in which $\mathbf{A}_{2}$ can be computed explicitly as

$$
\mathbf{A}_{2}=\left[\begin{array}{ccccc}
A_{e} & 0 & 0 & 0 & 0 \\
0 & \left(1+\mu^{2}\right) J_{x} & 0 & -2 \mu J_{x y} & 0 \\
0 & 0 & A_{e} & 0 & 0 \\
0 & -2 \mu J_{x y} & 0 & \left(1+\mu^{2}\right) J_{y} & 0 \\
0 & 0 & 0 & 0 & A_{e}
\end{array}\right],
$$


where (14) is used again, $J_{x}$ and $J_{y}$ are defined in (31), and $J_{x y}$ denotes the following integration:

$$
J_{x y}=\iint_{A_{e}} x y d x d y
$$

Therefore, the inverse of $\mathbf{A}_{2}$ can also be given explicitly as

$$
\mathbf{A}_{2}^{-1}=\left[\begin{array}{cc}
\frac{1}{A_{e}} & 0 \\
0 & \frac{\left(1+\mu^{2}\right) J_{y}}{\left(\left(1+\mu^{2}\right)^{2} J_{x} J_{y}-4 \mu^{2} J_{x y}{ }^{2}\right)} \\
0 & 0 \\
0 & \frac{2 \mu J_{x y}}{\left(\left(1+\mu^{2}\right)^{2} J_{x} J_{y}-4 \mu^{2} J_{x y}{ }^{2}\right)} \\
0 & 0
\end{array}\right.
$$

$$
\left.\begin{array}{ccc}
0 & 0 & 0 \\
0 & \frac{2 \mu J_{x y}}{\left(\left(1+\mu^{2}\right)^{2} J_{x} J_{y}-4 \mu^{2} J_{x y}^{2}\right)} & 0 \\
\frac{1}{A_{e}} & 0 & 0 \\
0 & \frac{\left(1+\mu^{2}\right) J_{x}}{\left(\left(1+\mu^{2}\right)^{2} J_{x} J_{y}-4 \mu^{2} J_{x y}^{2}\right)} & 0 \\
0 & 0 & \frac{1}{A_{e}}
\end{array}\right] .
$$$$
\frac{1}{A_{e}}
$$$$
0
$$

A substitution of (44) into (38) yields the element stiffness matrix $\mathbf{K}_{2 e}$ of QCQ4-2 as

$$
\mathbf{K}_{2 \mathbf{e}}=\mathbf{C}^{T} \mathbf{A}_{2}^{-1} \iint_{A_{e}} \mathbf{P}_{\mathbf{2}}^{T} \mathbf{D} \mathbf{P}_{\mathbf{2}} d x d y \mathbf{A}_{2}^{-1} \mathbf{C},
$$

where the matrix $\mathbf{D}$ for linear isotropic material is given in (37). The integration in (48) involving lower-order polynomials only can be carried out easily in the element local Cartesian coordinates. Consequently, the element stiffness matrix $\mathbf{K}_{2 e}$ of QCQ4-2 is also evaluated explicitly.

\section{Numerical Examples}

A number of standard benchmark problems proposed for membrane elements are solved using the four-node quadrilateral quasi-conforming membrane elements QCQ4-1 and QCQ4-2 presented in previous section to evaluate their reliability and accuracy. It should be pointed out that all the dimensions and material properties of the problems solved in this section are dimensionless as they appear in the literature in order that the present numerical results can be directly compared with those reported in the literature.

5.1. Verification of the Coordinate Invariance of the Assumed Strain Interpolations. The assumed in-plane strain field defined in (18) for QCQ4-1 and that defined in (43) for QCQ4-2 are not a complete linear polynomial. However, the strain interpolation defined in (43) is corresponding to the complete quadratic interpolations given in (41) and (42) for the in-plane displacement filed of the four-node quadrilateral plane element depicted in Figure 2. The strain interpolation in (18) for QCQ4-1 is corresponding to the in-plane displacement field given in (41) and (42) with a zero value of Poisson ratio. Since the sufficient condition of the coordinate invariance of the trial displacement fields for displacementbased elements is that the trial function of displacement interpolation is a complete polynomial up to the given order [1]. Therefore, both QCQ4-1 and QCQ4-2 theoretically should be coordinate invariant. Several numerical examples were carried out to verify the coordinate invariance of the resulting elements QCQ4-1 and QCQ4-2, and the numerical results approve the aforementioned theoretical analysis on the coordinate invariance of QCQ4-1 and QCQ4-2.

5.2. Patch Test for Membrane Elements. The standard membrane patch test suggested by Macneal and Harder [33] is considered here. The geometry properties of a rectangular panel with uniform thickness are the length $a=0.24$, the width $b=0.12$, and the thickness $t=0.001$. The material properties of the panel are the Young's modulus $E=10$ and Poisson ratio $\mu=0.25$. The patch composed of five irregular quadrilateral elements is depicted in Figure 3, and the dimensionless coordinates of the internal nodal points are listed in Table 1.

A linear displacement field for both of $u$ and $v$ defined in (49) is imposed on the external nodes of the patch. Both QCQ4-1 and QCQ4-2 yield the exact displacement values at the internal nodes of the patch. Therefore, both QCQ4-1 and QCQ4-2 pass the strict membrane patch test

$$
u(x, y)=x+\frac{y}{2}, \quad v(x, y)=y+\frac{x}{2} .
$$

To evaluate the accuracy of the present quadrilateral membrane elements, a number of quadrilateral membrane elements are used for the comparison study. The basic concepts in the element formulation of these reference elements are summarized in Table 2 . These four-node quadrilateral membrane elements are grouped into three categories based on whether independent internal parameters or drilling degrees of freedom are used. In Table 2, Q4-like elements refer to the four-node quadrilateral membrane elements with four corner nodes only, Q6-like elements represent the four-node quadrilateral membrane elements with two displacement-like internal parameters, and Q4-like elements with drilling DOF are the four-node quadrilateral membrane elements with four drilling degrees of freedom at the corner nodes. 


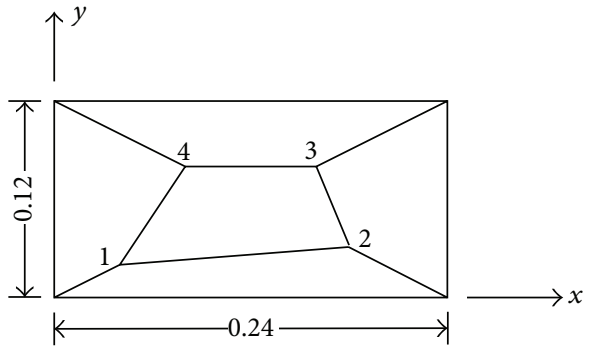

Figure 3: The mesh used for the patch test of membrane elements.

TABLE 1: The coordinates of the internal nodes.

\begin{tabular}{lcc}
\hline Node number & \multicolumn{3}{c}{ Coordinates } \\
\hline 1 & 0.04 & $y$ \\
2 & 0.18 & 0.02 \\
3 & 0.16 & 0.03 \\
4 & 0.08 & 0.08 \\
\hline
\end{tabular}

5.3. Cantilevered Beam Subjected to Transverse Load at the Free End with Regular Mesh. The cantilevered beam shown in Figure 4 is, respectively, divided by one regular quadrilateral element depicted in Figure 4 and five regular quadrilateral elements shown in Figure 5. The Yong's modulus $E=1500$, and the Poisson ratio $\mu=0.25$. The transverse forces are applied at three nodes as illustrated in Figure 4.

The results of the vertical displacement $V_{A}$ at point $A$ and the stress $\sigma_{x B}$ at point $B$ given by QCQ4-1 and QCQ42 are listed in Table 3. Some results obtained from other quadrilateral membrane elements are also given in the table for comparison. The information of the reference elements used in Table 3 is summarized in Table 2. The mesh $2 \times$ 10 is the mesh with two layers of elements along the beam thickness, and the stresses given by $2 \times 10$ mesh are at the same location as illustrated in Figure 5. The results of Q8 in Table 3 were computed using the popular CAE code ANSYS by the authors. It was shown by Shi and Voyiadjis [34] as well as Wang and Shi [35] that the analytical solution of the shear flexible cantilevered beam given in the text book of elasticity is not the exact solution. The numerical results of the fine mesh given by ANSYS-Q8 confirm the point given in $[34,35]$.

It can be seen from the table that both QCQ4-1 and QCQ4-2 give excellent results, and the inclusion of the Poisson ratio in the assumed strain field in QCQ4-2 improves the stress accuracy. This is because the assumed axial strain $\widetilde{\varepsilon}_{x}$ in an element of QCQ4-1 is constant, while the axial strain in QCQ4-2 is linearly varying as shown in (41). One can see that the performance of both QCQ4-1 and QCQ4-2 can match the Q4-like membrane elements with drilling degrees of freedom.

\subsection{Cantilevered Beam Subjected to Different Loads under} Irregular Mesh. This example is used to evaluate the computational accuracy of membrane elements to mesh irregularity. The cantilevered beam shown in Figure 4 is meshed by

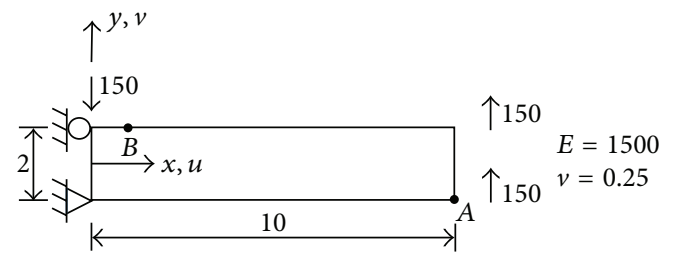

Figure 4: A cantilevered beam subjected to transverse load.

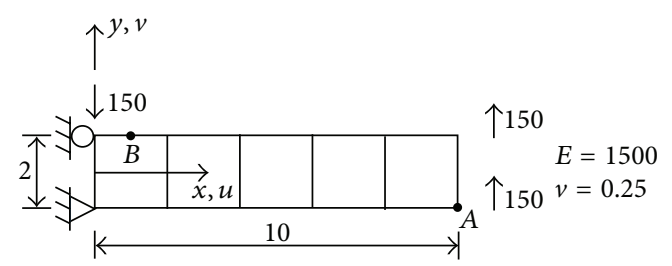

FiguRE 5: A $1 \times 5$ regular mesh for the cantilevered beam.

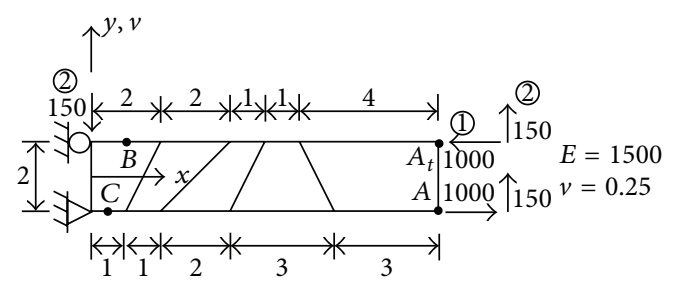

FIGURE 6: The irregular $1 \times 5$ regular mesh for the cantilevered beam.

five irregular quadrilateral membrane elements depicted in Figure 6. Two cases of loading are considered, Load 1 is the force couple acting at the two corners on the free end, and Load 2 is two equal transverse forces acting at the two corners. The numerical results given by QCQ4-1 and QCQ42 are tabulated in Table 4 together with the results given by a number of other popular quadrilateral membrane elements for comparison.

The results in Table 4 show that QCQ4-1 and QCQ42 deliver very accurate results for both displacements and stresses, especially when the Poisson effect is taken into account. Furthermore, the comparison of the results in Table 4 indicates that the displacement given by QCQ4-1 and QCQ4-2 can match the accuracy of both the Q6-type membrane elements and the four-node membrane elements with drilling degrees of freedom. One can also see that the predicted stresses by the present elements are much better than the displacement-based Q6-type elements.

Most of researchers only examined the accuracy of the computed deflection at the bottom corner at the free end of the cantilevered beam illustrated in Figure 6. However, it is interesting and worthwhile to check the deformation pattern of the cantilever predicted by four-node membrane elements. To this end, the deflections $v_{A}$ and $v_{A t}$, respectively, at the lower-right corner $A$ and the upper-right corner $A_{t}$ of the cantilever shown in Figure 6, obtained from the five-beta hybrid stress elements summarized in [32] and by the present five strain parameter elements are tabulated in Table 5 , in which $\kappa$ appearing in some elements is a scaling parameter 
TABLE 2: List of element models for comparison study.

\begin{tabular}{|c|c|c|c|}
\hline No. & Symbols & Formulation methods & References \\
\hline 1 & Q4 & 4-node isoparametric membrane element & [5] \\
\hline 2 & Q6 & 4-node isoparametric element with 2 internal parameters & [5] \\
\hline 3 & QM6 & Modified Q6 element & {$[5]$} \\
\hline 4 & QC6 & Q6-like quasi-conforming isoparametric element & {$[5,21]$} \\
\hline 5 & PS & Q4-like hybrid stress element & {$[12]$} \\
\hline 6 & $07 \beta$ & Q4-like element with drilling dof using hybrid stress method & {$[10]$} \\
\hline 7 & QR4b & Q4-like element with drilling dof using the quasi-conforming element technique & {$[7]$} \\
\hline 8 & QA4 & Q4-like element with drilling dof using the quasi-conforming element technique & {$[8]$} \\
\hline 9 & I-A & Q4-like element with drilling dof based on a special functional & {$[9]$} \\
\hline 10 & RQ4 & Q4 element based on rational displacement field & {$[26]$} \\
\hline 11 & AQCE4 & Q4-like quasi-conforming element based on an assumed rational strain field & {$[26]$} \\
\hline 12 & B-Q4E & Q4-like assumed strain element based on B-bar method & {$[17,18]$} \\
\hline 13 & Pimpinelli & Q4-like assumed strain element with drilling dof & {$[15]$} \\
\hline 14 & CYSE & Q4-like element by natural strain with 7 enhancing variables for membrane strains & {$[6]$} \\
\hline 15 & QACII6 & Q6-like element based on quadrilateral area coordinates & {$[18]$} \\
\hline 16 & AGQ6-I & Q6-like element based on quadrilateral area coordinates & {$[5,17]$} \\
\hline 17 & AGQ6M & Modified AGQ6-I element to pass patch test & {$[5,17]$} \\
\hline 18 & WDQ4 & Q4-like quasi-conforming element with bilinear interpolation for element domain displacement & {$[28]$} \\
\hline 19 & WDQ4 & Q4-like quasi-conforming element with complete quadratic interpolation for element domain displ. & [28] \\
\hline
\end{tabular}

TABLE 3: Displacements and stresses given by various elements with regular meshes.

\begin{tabular}{|c|c|c|c|c|c|c|}
\hline \multirow{2}{*}{ Elements } & \multicolumn{2}{|c|}{$1 \times 1$} & \multicolumn{2}{|c|}{$1 \times 5$} & \multicolumn{2}{|c|}{$2 \times 10$} \\
\hline & $v_{A}$ & $\sigma_{x B}$ & $v_{A}$ & $\sigma_{x B}$ & $v_{A}$ & $\sigma_{x B}$ \\
\hline $\mathrm{QA}^{1}$ & 77.5 & 2250.0 & 101.51 & 4049.88 & - & - \\
\hline $\mathrm{QR}_{4} \mathrm{~b}^{1}$ & 75.5 & 2250.0 & 102.64 & 4049.90 & - & - \\
\hline RQ4 & 79.62 & 2025 & 104.45 & 4050 & - & - \\
\hline WDQ4 & - & - & 70 & - & 91.476 & - \\
\hline WDQ6 & - & - & 70 & - & 91.450 & - \\
\hline QCQ4-1 & 72.81 & 2227.5 & 95.31 & 4050.0 & 100.25 & 4232.25 \\
\hline QCQ4-2 & 77.50 & 3037.5 & 101.50 & 4050.0 & 102.00 & 4262.22 \\
\hline Analytical & 102.6 & 4050 & 102.6 & 4050 & 102.6 & 4050 \\
\hline ANSYS-Q8 & 102.04 & - & 102.12 & 4050 & 102.92 & 4056.0 \\
\hline
\end{tabular}

${ }^{1}$ With drilling degrees of freedom.

used to immunize the trapezoidal locking [32]. The predicted tensile and compressive stresses in the element with the fixed end are also listed in the table.

The results in Table 5 show that all these five-beta hybrid stress membrane elements give very accurate stresses, particularly the elements with the larger scaling parameter $\kappa$. However, the difference of $\left(v_{A}-v_{A t}\right)$, which is the deflection difference between the two corners along the free edge, given by all these the five-beta hybrid stress elements, is also very large. Moreover, the larger $\kappa$ is, the larger deflection difference is. The smallest $\left(v_{A}-v_{A t}\right)$ yielded by these hybrid stress elements is 2.17 given by PS, which also has a difference larger than 2.2\%. $v_{A}$ and $v_{A t}$ given by QCQ4-1 and QCQ42 are not identical either although the deflection difference is smaller. The larger deflection difference along the free edge implies that the cantilever-like membrane under the bending and shearing loading along the free edge illustrated in Figure 6 undergoes a considerable tensile deformation in the transverse direction. As shown by the analytical solutions in the table, there is no tensile deformation along the free edge of the cantilever-like membrane since the deflections along the free edge are identical. Then a questions arises: does the deformation of the two-dimensional cantilevered beam subjected to bending load computed by these membrane elements with the mesh shown in Figure 6 make sense? Or put the question in another way: can the membrane with bending as the dominant deformation illustrated in Figure 6 be meshed with only one-layer irregular membrane elements along its transverse direction? The authors think that this question deserves further discussion.

5.5. Cook's Membrane. This popular benchmark problem was proposed by Cook [36]. The geometry and the material parameters of the membrane are shown in Figure 7, and 
TABLE 4: Element performance under $1 \times 5$ irregular mesh for a cantilevered beam.

\begin{tabular}{|c|c|c|c|c|c|c|}
\hline \multirow{2}{*}{ Element symbol } & \multirow{2}{*}{ Formation of $\mathbf{K}_{e}$} & \multirow{2}{*}{ Patch test } & \multicolumn{2}{|c|}{ Load 1} & \multicolumn{2}{|c|}{ Load 2} \\
\hline & & & $v_{A}$ & $\sigma_{x B}$ & $v_{A}$ & $\sigma_{x B}$ \\
\hline $\mathrm{Q} 4$ & Numer. Integr. & Pass & 45.7 & -1761 & 50.7 & -2448 \\
\hline Q6 & Numer. Integr. & Fail & 98.4 & -2428 & 100.4 & -3354 \\
\hline QM6 & Numer. Integr. & Pass & 96.1 & -2497 & 98.0 & -3235 \\
\hline QC6 & Numer. Integr. & Pass & 96.1 & -2439 & 98.1 & -3339 \\
\hline PS & Numer. Integr. & Pass & 96.2 & -3014 & 98.19 & -4137 \\
\hline $\mathrm{QR}_{4} \mathrm{~b}^{1}$ & Numer. Integr. & Pass & 100.4 & -3026.0 & 101.5 & -3798.4 \\
\hline QA4 ${ }^{1}$ & Numer. Integr. & Pass & 97.4 & -2981.6 & 98.9 & -4077.8 \\
\hline $07 \beta^{1}$ & Explicit & Pass & 100.1 & -2997 & 102.2 & -4131 \\
\hline RQ4 & Explicit & Pass & - & - & 84.0 & -3746 \\
\hline AQCE4 & Explicit & Pass & - & - & 97.2 & -4119 \\
\hline B-Q4E & Numer. Integr. & n.a. & 96.5 & -3004 & 98.3 & -3906 \\
\hline QACII6 & Numer. Integr. & Fail & 100 & -3000 & 102.7 & -4180 \\
\hline AGQ6-I & Numer. Integr & Fail & 100.0 & -3000 & 102.0 & -4151 \\
\hline AGQ6M & Numer. Integr & Pass & 96.0 & -3015 & 97.9 & -4135 \\
\hline QCQ4-1 & Explicit & Pass & 89.3 & -2979 & 93.1 & -4178 \\
\hline QCQ4-2 & Explicit & Pass & 94.6 & -2958 & 99.1 & -4123 \\
\hline Analytical & - & - & 100.0 & -3000 & 102.6 & -4050 \\
\hline ANSYS-Q8 & $2 \times 10$ mesh & - & 101.4 & -3000.0 & 102.9 & -4056.0 \\
\hline
\end{tabular}

${ }^{1}$ With drilling degrees of freedom.

TABLE 5: The nodal deflections at the free end and stresses in the element with the fixed end of a cantilevered beam with $1 \times 5$ irregular mesh.

\begin{tabular}{lcccc}
\hline Elements & $v_{A}$ & $v_{A t}$ & $\sigma_{x C}$ & $\sigma_{x B}$ \\
\hline PS & 96.18 & 94.01 & 3007 & -3014 \\
YHP [30] & 96.50 & 94.34 & 3006 & -3013 \\
PS-E [31] & 99.88 & 97.57 & 3000 & -3000 \\
SPS $(\kappa=500)[32]$ & 101.79 & 97.33 & 3001 & -3003 \\
SYHP $(\kappa=500)[32]$ & 101.78 & 97.37 & 3001 & -3002 \\
SPS $(\kappa=5000)[32]$ & 102.10 & 97.54 & 3000 & -3000 \\
SYHP $(\kappa=5000)[32]$ & 102.10 & 97.54 & 3000 & -3000 \\
QCQ4-1 & 89.33 & 88.64 & 2983.5 & -2979.3 \\
QCQ4-2 & 94.59 & 94.03 & 2969.0 & -2958.3 \\
Analytical solutions & 100 & 100 & 3000 & -3000 \\
\hline
\end{tabular}

this membrane is clamped at its left edge and subjected to distributed shear load along its left edge.

Three different meshes densities shown in Table 6 are used to compute the displacement and stress of the membrane. This skew membrane presents the typical features of the in-plane deformation of plane stress problems in theory of elasticity as the in-plane bending is not the dominant deformation. Therefore, it is a suitable membrane problem to be solved by membrane elements using coarse meshes and it can serve as a good benchmark for the accuracy comparison of membrane elements in real engineering problems.

The vertical displacement at the middle point of the free edge and the maximum stress at the middle of the bottom edge given by QCQ4-1 and QCQ4-2 and other quadrilateral membrane elements are tabulated in Table 6 . The reference

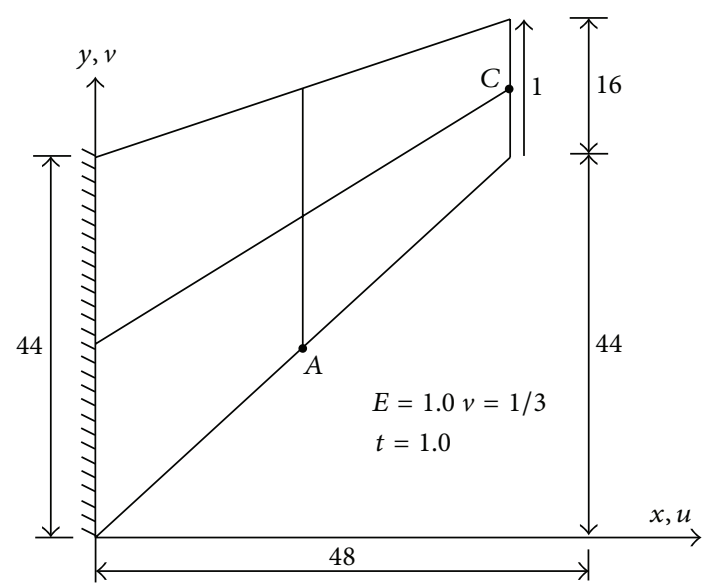

Figure 7: The geometry and material properties of Cook's membrane.

displacement and stress for this problem are taken from [7]. The results in Table 6 clearly demonstrate again that the accuracy of both the displacement and stress evaluated by the present elements QCQ4-1 and QCQ4-2 can match all the improved Q6-type elements, all the Q6-like elements formulated by hybrid stress methods or other assumed strain methods, and all the quadrilateral membrane elements with drilling degrees of freedom, although the computational efficiency of QCQ4-1 and QCQ4-2 is much higher than all of these sophisticated quadrilateral membrane elements.

5.6. MacNeal's Slender Beams. The slender cantilevered beam with rectangular cross-section depicted in Figure 8 was proposed to test the trapezoidal locking of membrane elements 
TABLE 6: The results of Cook's membrane given by different membrane elements.

\begin{tabular}{|c|c|c|c|c|c|c|}
\hline \multirow{2}{*}{ Elements } & \multicolumn{2}{|c|}{$2 \times 2$ mesh } & \multicolumn{2}{|c|}{$4 \times 4$ mesh } & \multicolumn{2}{|c|}{$8 \times 8$ mesh } \\
\hline & $v_{C}$ & $\sigma_{A \max }$ & $v_{C}$ & $\sigma_{A \max }$ & $v_{C}$ & $\sigma_{A \max }$ \\
\hline Q4 & 11.80 & 0.1217 & 18.29 & 0.1837 & 22.08 & 0.2242 \\
\hline Q6 & 22.94 & 0.2029 & 23.48 & 0.2258 & 23.80 & 0.2334 \\
\hline QM6 & 21.05 & 0.1928 & 23.02 & 0.2243 & - & - \\
\hline QC6 & 21.13 & - & 23.02 & - & - & - \\
\hline PS & 21.13 & 0.1855 & 23.02 & 0.2242 & - & - \\
\hline $\mathrm{QR}_{4 \mathrm{~b}^{1}}$ & 22.48 & 0.2074 & 23.43 & 0.2337 & - & - \\
\hline $\mathrm{QA}^{1}$ & 23.29 & 0.1883 & 23.78 & 0.2226 & - & - \\
\hline I- $A^{1}$ & 23.37 & - & 23.90 & - & 23.99 & - \\
\hline $07 \beta^{1}$ & 22.586 & 0.1971 & 23.446 & 0.2318 & - & - \\
\hline RQ4 & 19.79 & 0.1902 & 22.93 & 0.2303 & - & - \\
\hline AQCE4 & 22.32 & 0.1414 & 24.12 & 0.1928 & - & - \\
\hline B-Q4E & 21.35 & 0.1956 & 23.04 & 0.2261 & - & - \\
\hline $\mathrm{CYSE}^{2}$ & 10.40 & - & 17.65 & - & 21.90 & - \\
\hline Pimpinelli ${ }^{1}$ & 21.02 & - & 23.01 & - & 23.68 & - \\
\hline QACII6 & 25.92 & 0.2169 & 24.37 & 0.2286 & 24.04 & 0.2352 \\
\hline AGQ6-I & 23.06 & 0.2023 & 23.68 & 0.2275 & 23.87 & 0.2351 \\
\hline AGQ6M & 20.74 & - & 22.99 & - & 23.69 & - \\
\hline WDQ4 & 16.35 & - & 21.35 & - & 23.17 & - \\
\hline QCQ4-1 & 22.07 & 0.2331 & 23.44 & 0.2504 & 23.78 & 0.2393 \\
\hline QCQ4-2 & 23.09 & 0.2207 & 23.59 & 0.2350 & 23.82 & 0.2327 \\
\hline Reference & 23.90 & 0.2360 & 23.90 & 0.2360 & 23.90 & 0.2360 \\
\hline
\end{tabular}

${ }^{1}$ With drilling degrees of freedom.

${ }^{2}$ The value read from the curves shown in the figures.

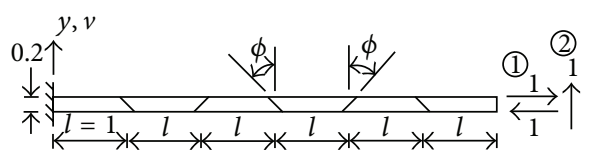

FIGURE 8: MacNeal's slender beam modeled by a layer of trapezoidal plane elements.

$[5,32]$. Two cases of bending loads are considered here as in Section 5.4. The width of the beam is $t=0.1$. The Young's modulus of the beam is $E=10^{7}$, and the Poisson ratio is $\mu=0.3$ The dimensionless exact deflections at the free end are 0.0054 and 0.1081 corresponding, respectively, to Load 1 and Load 2 [5].

A single layer of membrane elements is used to model the slender beam as shown in Figure 8. Three angles of $\phi$ for the trapezoidal elements are considered here to check the sensitivity of mesh distortion in the bending analysis of the beam. The deflections at the beam-free end given by different membrane elements are tabulated in Table 7, where the results in the first five rows are taken from [5].

The beam shown in Figure 8 undergoes bending deformation under the action of the loading shown in Figure 8. It can be seen from the results in Table 7 that Q4 suffers severe shear locking even in the case of rectangular elements, AGQ6-I [5] yields excellent results for all cases, and SPS and SYHP can give good results when very large scaling parameters are used [32]. QCQ4-2, which accounts for the curvature by the Poisson effect shown in (43), gives good results under the regular elements for both of the loading cases, but it cannot characterize the curvatures of beams well in the case of trapezoidal elements. In general, QCQ4-1 and QCQ4-2 are sensitive to the mesh distortion when they are used in the analysis of the beam-like membranes illustrated in Figure 8 where the bending is the dominant deformation and only a single layer of membrane elements is used. However, it is once again one would ask that whether it is feasible to use only one layer of irregular membrane elements to model the bending problems of beams.

\section{Conclusions}

Based on the quasi-conforming element technique, two fournode quadrilateral membrane elements with eight nodal displacement parameters totally, designated as QCQ4-1 and QCQ4-2, respectively, are presented in this paper. The difference between these quasi-conforming membrane elements is that the Poisson effect is included explicitly in the assumed strain fields of QCQ4-2. The element formulations and numerical results show that the present four-node quadrilateral membrane elements have the following features.

(1) The present four-node quadrilateral quasi-conforming membrane elements QCQ4-1 and QCQ4-2 derived by 5 strain parameters are reliable and robust as they pass the patch test and are free from shear 
TABLE 7: The normalized deflections at the free end of the MacNeal's slender beam by using one layer of different trapezoidal membrane elements.

\begin{tabular}{|c|c|c|c|c|c|c|}
\hline \multirow{2}{*}{ Element model } & \multicolumn{2}{|c|}{$\phi=0^{\circ}$} & \multicolumn{2}{|c|}{$\phi=\arctan (1 / 2)$} & \multicolumn{2}{|c|}{$\phi=45^{\circ}$} \\
\hline & Load 1 & Load 2 & Load 1 & Load 2 & Load 1 & Load 2 \\
\hline Q4 & 0.093 & 0.093 & - & - & 0.022 & 0.003 \\
\hline Q6 & 1.000 & 0.993 & - & - & 0.093 & 0.106 \\
\hline QM6 & 1.000 & 0.993 & - & - & 0.037 & 0.044 \\
\hline AGQ6-I & 1.000 & 0.993 & - & - & 1.000 & 0.994 \\
\hline AGQ6M & 1.000 & 0.993 & - & - & 0.046 & 0.051 \\
\hline PS & - & - & 0.117 & 0.133 & 0.046 & 0.052 \\
\hline YHP [30] & - & - & 0.117 & 0.133 & 0.047 & 0.518 \\
\hline PS-E [31] & - & - & 0.950 & 0.951 & 0.955 & 0.955 \\
\hline SPS $(\kappa=50)[32]$ & - & - & 0.415 & 0.494 & 0.394 & 0.476 \\
\hline $\operatorname{SYHP}(\kappa=50)[32]$ & - & - & 0.416 & 0.495 & 0.398 & 0.480 \\
\hline SPS $(\kappa=5000)[32]$ & - & - & 0.983 & 0.978 & 0.983 & 0.978 \\
\hline SYHP $(\kappa=5000)[32]$ & - & - & 0.983 & 0.978 & 0.983 & 0.978 \\
\hline QCQ4-1 & 0.910 & 0.904 & 0.107 & 0.134 & 0.026 & 0.074 \\
\hline QCQ4-2 & 1.000 & 0.993 & 0.110 & 0.139 & 0.053 & 0.077 \\
\hline
\end{tabular}

locking and insensitive to the element distortion in the analysis of practical plane elasticity problems.

(2) These two quasi-conforming membrane elements are very accurate although the element formulation is extremely simple, as no nonconforming mode as well as no mapping of the natural coordinates is involved. It is worthwhile to emphasize that these quasi-conforming elements yield not only accurate displacements and also very accurate stress evaluation.

(3) These two quasi-conforming quadrilateral membrane elements are very computationally efficient as the element stiffness matrix is given explicitly in Cartesian coordinates; that is, there is no any coordinate mapping and numerical integration employed at all in the computation of element stiffness matrix. It can be concluded that the membrane elements presented in this paper are the simplest shear locking free quadrilateral membrane elements reported in the literature to date.

(4) The present quasi-conforming membrane elements are not only simpler in formulation but also more accurate than other quasi-conforming quadrilateral membrane elements where different methods are used to evaluate the strain parameters of the assumed element strain field.

(5) The performance study of QCQ4-2 shows that the explicit inclusion of Poisson effect in the assumed strain field can improve the accuracy when the coarse meshes are used.

The four-node quadrilateral membrane elements presented in this paper provide reliable, efficient, and accurate elements for the displacement and stress analysis of plane stress and plane strain problems as well as the analysis of shell-like structures based on flat-shell elements.

\section{Conflict of Interests}

The authors declare that there is no conflict of interests regarding the publication of this paper.

\section{Acknowledgment}

The financial support provided by the Grant of NSFC91230113 is thankfully acknowledged.

\section{References}

[1] O. C. Zienkewich, R. L. Taylor, and J. Z. Zhu, The Finite Element Method: Its Basis \& Fundamentals, Elsevier, Singapore, 6th edition, 2009.

[2] G. Shi and G. Z. Voyiadjis, "Geometrically nonlinear analysis of plates by assumed strain element with explicit tangent stiffness matrix," Computers \& Structures, vol. 41, no. 4, pp. 757-763, 1991.

[3] LS-DYNA Theory Manual (Version 971), Livermore Software Technology, Livermore, Calif, USA, 2006.

[4] E. L. Wilson, R. L. Taylor, W. P. Doherty, and T. Ghabussi, "Incompatible displacement models," in Numerical Computational Methods in Structural Mechanics, S. T. Fenven, Ed., pp. 43-57, Academic Press, New York, NY, USA, 1973.

[5] X.-M. Chen, S. Cen, Y.-G. Li, and J.-Y. Sun, "Several treatments on nonconforming element failed in the strict patch test," Mathematical Problems in Engineering, vol. 2013, Article ID 901495, 7 pages, 2013.

[6] R. P. R. Cardoso, J. W. Yoon, and R. A. F. Valente, "A new approach to reduce membrane and transverse shear locking for one-point quadrature shell elements: linear formulation," International Journal for Numerical Methods in Engineering, vol. 66, no. 2, pp. 214-249, 2006.

[7] H. Liu, L. Tang, and H. Lv, "The quasi-conforming plane elements with rotational degree of freedom," Computational Structural Mechanics and Applications, vol. 7, no. 4, pp. 23-31, 1990 (Chinese). 
[8] W. Chen and Y. Li, "Refined non-conforming quadrilateral plane isoparametric element with drilling degrees of freedom," Computational Structural Mechanics and Applications, vol. 10, no. 1, pp. 22-29, 1993.

[9] M. Iura and S. N. Atluri, "Formulation of a membrane finite element with drilling degrees of freedom," Computational Mechanics, vol. 9, no. 6, pp. 417-428, 1992.

[10] K. Y. Sze, W. Chen, and Y. K. Cheung, "An efficient quadrilateral plane element with drilling degrees of freedom using orthogonal stress modes," Computers \& Structures, vol. 42, no. 5, pp. 695-705, 1992.

[11] T. J. R. Hughes, F. A. Masud, and I. Harari, "Numerical assessment of some membrane elements with drilling degrees of freedom," Computers and Structures, vol. 55, no. 2, pp. 297314, 1995.

[12] T. H. H. Pian and K. Sumihara, "Rational approach for assumed stress finite elements," International Journal for Numerical Methods in Engineering, vol. 20, no. 9, pp. 1685-1695, 1984.

[13] R. Piltner and R. L. Taylor, "A systematic construction of Bbar functions for linear and non-linear mixed-enhanced finite elements for plane elasticity problems," International Journal for Numerical Methods in Engineering, vol. 44, no. 5, pp. 615-639, 1999.

[14] J. M. A. De César Sá, R. M. Natal Jorge, R. A. Fontes Valente, and P. M. Almeida Areias, "Development of shear locking-free shell elements using an enhanced assumed strain formulation," International Journal for Numerical Methods in Engineering, vol. 53, no. 7, pp. 1721-1750, 2002.

[15] G. Pimpinelli, "An assumed strain quadrilateral element with drilling degrees of freedom," Finite Elements in Analysis and Design, vol. 41, no. 3, pp. 267-283, 2004.

[16] W. Zhong and Z. Ji, "Rational finite element," Computational Structural Mechanics and Applications, vol. 13, no. 1, pp. 1-8, 1996 (Chinese).

[17] X.-M. Chen, S. Cen, Y.-Q. Long, and Z.-H. Yao, "Membrane elements insensitive to distortion using the quadrilateral area co-ordinate method," Computers and Structures, vol. 82, no. 1, pp. 35-54, 2004.

[18] X. M. Chen, S. Cen, X. R. Fu, and Y. Q. Long, "A new quadrilateral area coodinate method (QACM-II) for developing quadrilateral finite element models," International Journal for Numerical Methods in Engineering, vol. 73, no. 13, pp. 1911-1941, 2008.

[19] L. Tang, W. Chen, and Y. Liu, "The quasi-conforming element technique for finite element analysis," Journal of Dalian University of Technology, vol. 19, no. 2, pp. 19-35, 1980 (Chinese).

[20] W. Chen, Y. Liu, and L. Tang, "The formulation of quasiconforming elements," Journal of Dalian University of Technology, vol. 19, no. 2, pp. 37-50, 1980 (Chinese).

[21] W. Chen and L. Tang, "Isoparametric quasi-conforming element," Journal of Dalian University of Technology, vol. 20, no. 1, pp. 63-74, 1981 (Chinese).

[22] L. Tang, W. Chen, and Y. Liu, "String net function approximation and quasi-conforming technique," in Hybrid and Mixed Finite Element Methods, S. N. Atluri, R. H. Gallagher, and O. C. Zienkiewich, Eds., pp. 173-188, Wiley, New York, NY, USA, 1983.

[23] G. Shi and G. Z. Voyiadjis, "A Simple $C^{0}$ quadrilateral thick/thin shell element based on the refined shell theory and the assumed strain fields," International Journal of Solids \& Structures, vol. 27, no. 3, pp. 283-298, 1991.
[24] G. Shi and G. Z. Voyiadjis, "Efficient and accurate four-node quadrilateral $\mathrm{C}^{0}$ plate bending element based on assumed strain fields," International Journal for Numerical Methods in Engineering, vol. 32, no. 5, pp. 1041-1055, 1991.

[25] G. R. Lomboy, S. Suthasupradit, K.-D. Kim, and E. Oñate, "Nonlinear formulations of a four-node quasi-conforming shell element," Archives of Computational Methods in Engineering, vol. 16, no. 2, pp. 189-250, 2009.

[26] T. Liu, Y. Zhu, and H. Lu, "Analytical quasi-conformed plane quadrilateral element," Chinese Journal of Computational Mechanics, vol. 15, no. 3, pp. 324-328, 1998 (Chinese).

[27] G. Shi and G. Z. Voyiadjis, "Simple and efficient shear flexible two-node arch/beam and four-node cylindrical shell/plate finite elements," International Journal for Numerical Methods in Engineering, vol. 31, no. 4, pp. 759-776, 1991.

[28] Y. Xia, P. Hu, and L. M. Tang, "Direct formulation of quadrilateral plane element with quasi-conforming method into the forbidden zone of FEM," Chinese Journal of Theoretical and Applied Mechanics, vol. 44, no. 5, pp. 839-850, 2012 (Chinese).

[29] G. Shi and L. Tang, "Weak forms of generalized governing equations in theory of elasticity," Interaction and Multiscale Mechanics, vol. 1, no. 3, pp. 329-337, 2008.

[30] K.-Y. Yuan, Y.-S. Huang, and T. H. H. Pian, "New strategy for assumed stresses for 4-node hybrid stress membrane element," International Journal for Numerical Methods in Engineering, vol. 36, no. 10, pp. 1747-1763, 1993.

[31] C.-C. Wu and Y. K. Cheung, "On optimization approaches of hybrid stress elements," Finite Elements in Analysis and Design, vol. 21, no. 1-2, pp. 111-128, 1995.

[32] K. Y. Sze, "On immunizing five-beta hybrid-stress element models from 'trapezoidal locking' in practical analyses," International Journal for Numerical Methods in Engineering, vol. 47, no. 4, pp. 907-920, 2000.

[33] R. H. Macneal and R. L. Harder, "A proposed standard set of problems to test finite element accuracy," Finite Elements in Analysis and Design, vol. 1, no. 1, pp. 3-20, 1985.

[34] G. Shi and G. Z. Voyiadjis, "A sixth-order theory of shear deformable beams with variational consistent boundary conditions," Journal of Applied Mechanics, vol. 78, no. 2, Article ID 021019, 11 pages, 2011.

[35] X. D. Wang and G. Shi, “The boundary layer solutions induced by displacement boundary conditions of shear deformable beams and accuracy study of several higher-order beam theories," Journal of Engineering Mechanics, vol. 138, no. 11, pp. 13881399, 2012.

[36] R. D. Cook, "Improved two-dimensional finite element," ASCE Journal of the Structural Division, vol. 100, pp. 1851-1865, 1974. 


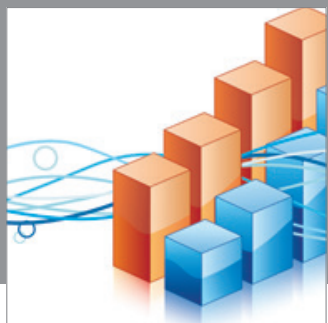

Advances in

Operations Research

mansans

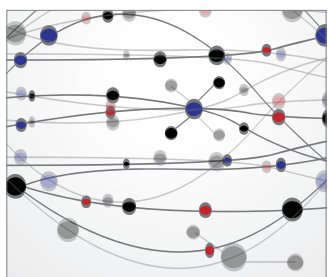

The Scientific World Journal
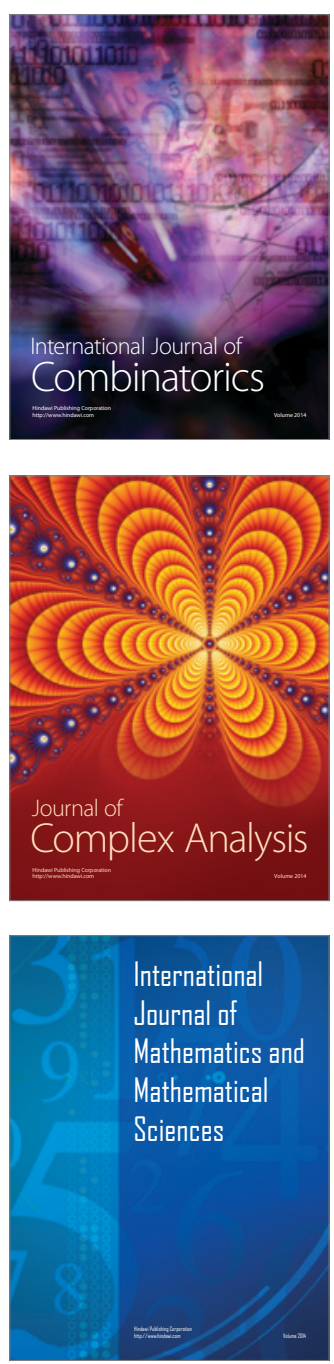
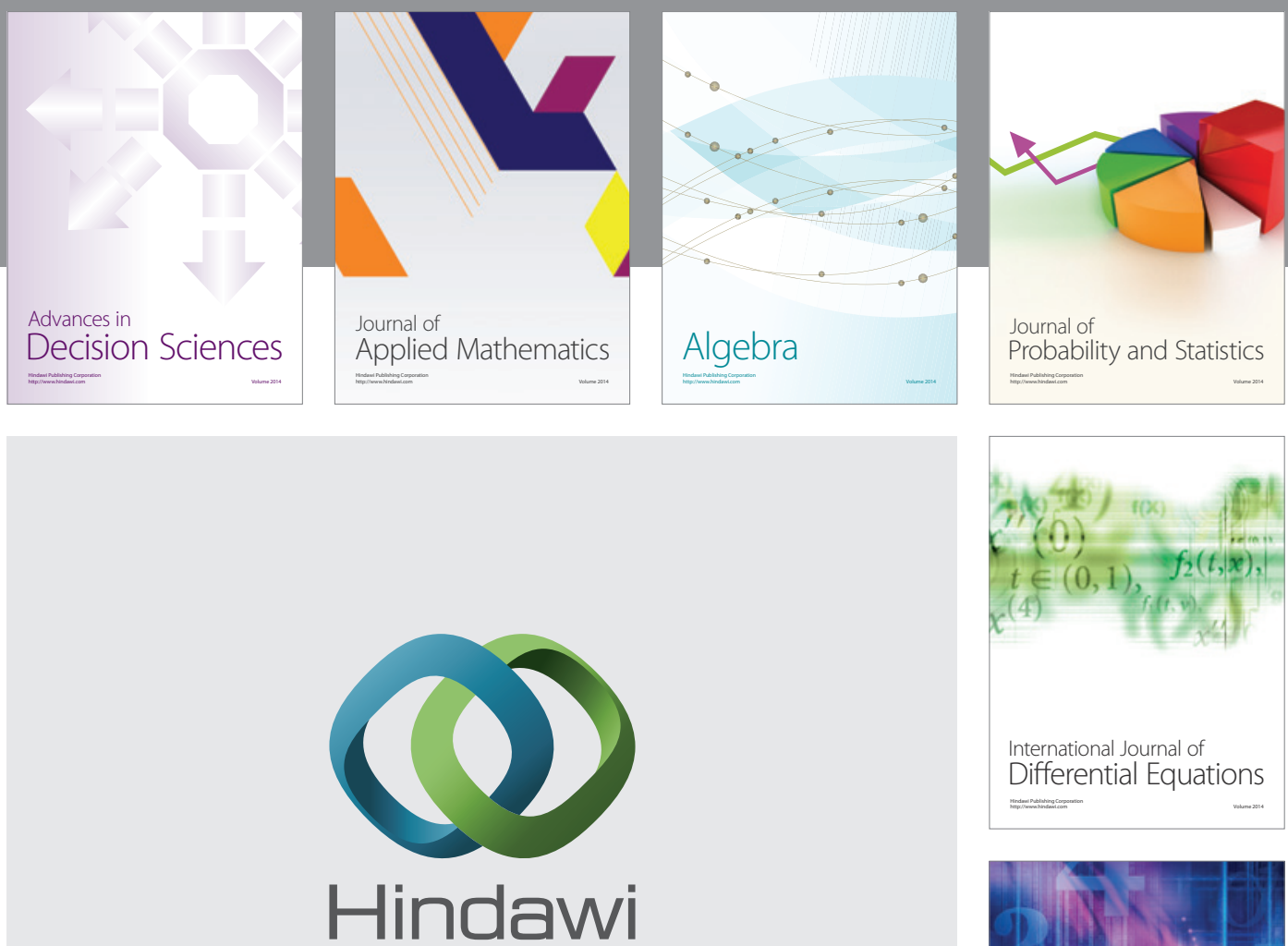

Submit your manuscripts at http://www.hindawi.com
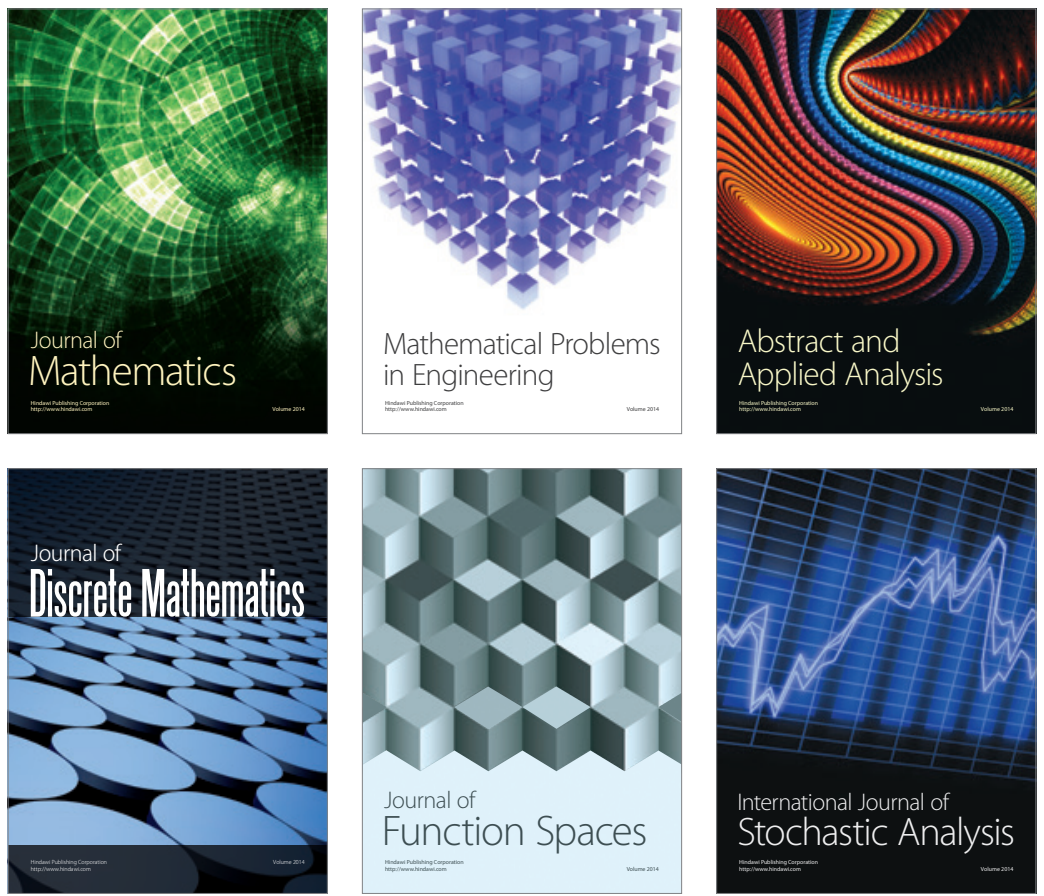

Journal of

Function Spaces

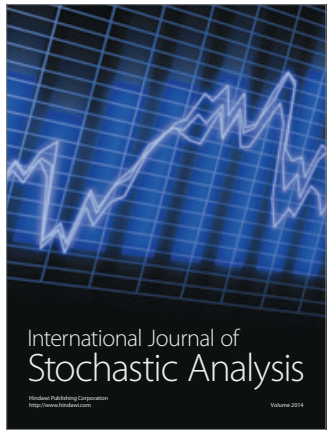

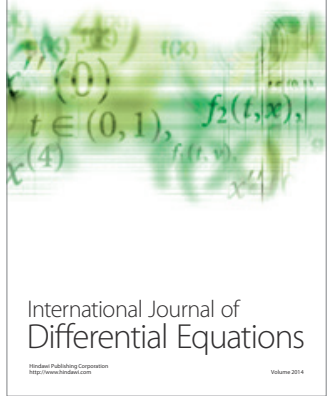
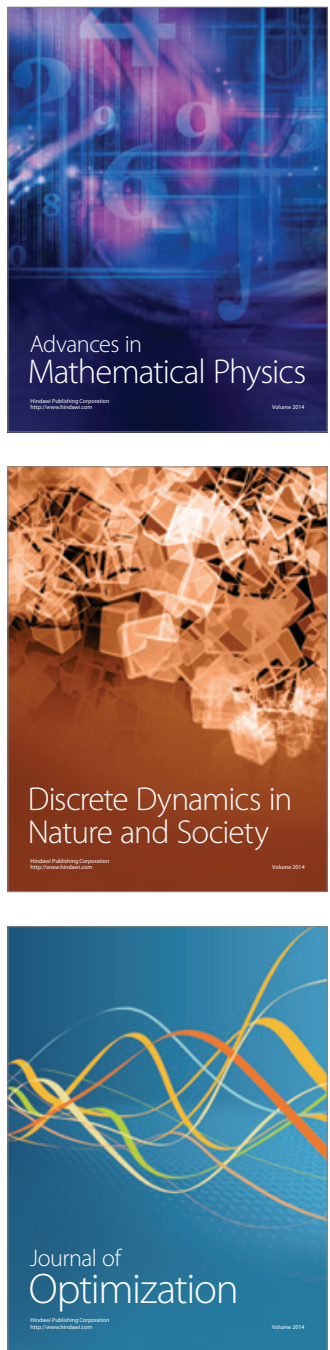